\title{
Relato de experiência sobre a percepção da influência da agroecologia no bem-estar de horticultores rurais através de projeto sistematizador
}

\author{
Experience report on the perception of the influence of agroecology on the well-being of rural \\ horticulturists through a systematic project \\ Informe de experiencia sobre la percepción de la influência de la agroecología en el bienestar de los \\ horticultores rurales a través de un proyecto sistemático
}

\author{
Fernanda da Silva Pereira \\ ORCID: https://orcid.org/0000-0001-6243-2480 \\ Universidade Federal de Rondônia, Brasil \\ Email:nanda31101989@gmail.com
}

\begin{abstract}
Resumo
A agroecologia surge como um fulgor à vida de agricultores rurais, a sua base em produção sustentável pode garantir uma maior qualidade de vida a população rural. Este relato de experiência procura expor percepções obtidas através de um trabalho desenvolvido com um grupo de agricultores agroecológicos, moradores em uma comunidade da zona rural do município de Cacoal-RO. O objetivo é relatar uma experiência cujos dados foram coletados através da vivência e observação sobre a influência que as práticas agroecológicas apresentaram na melhoria do bem-estar dos agricultores e assim contribuir com futuras pesquisas que acresça a capilaridade das ações agroecológicas. O grupo de agricultores é composto por 10 famílias que trabalham diretamente com agroecologia e participam das atividades ativamente. O trabalho de acompanhamento do grupo foi de aproximadamente 12 meses. As percepções analisada neste relato se deram simultaneamente ao processo de sistematização de práticas agroecológicas do grupo Bem Viver, atividades que faziam parte da execução de um projeto de mestrado. Conclui-se que o trabalho da horta exerceu forte influência na melhoria da autonomia e bem-estar dos integrantes do grupo.
\end{abstract}

Palavras-chave: Agroecologia; Sistematização de experiência; Bem estar do horticultor rural.

\begin{abstract}
Agroecology appears as a glow to the life of rural farmers, its base in sustainable production can guarantee a better quality of life for the rural population. This experience report seeks to expose perceptions obtained through work developed with a group of agroecological farmers, living in a community in the rural area of the municipality of CacoalRO. The objective is to report an experience whose data were collected through experience and observation about the influence that agroecological practices had on improving the well-being of farmers and thus contributing to future research that adds to the capillarity of agroecological actions. The group of farmers is composed of 10 families who work directly with agroecology and actively participate in the activities. The group's follow-up work was approximately 12 months. The perceptions analyzed in this report occurred simultaneously with the process of systematizing agroecological practices of the Bem Viver group, activities that were part of the execution of a master's project. It is concluded that the work of the vegetable garden had a strong influence in improving the autonomy and well-being of the members of the group.
\end{abstract}

Keywords: Agroecology; Systematization of experience; Well-being of the rural horticulturist.

\section{Resumen}

La agroecología aparece como un resplandor a la vida de los agricultores rurales, su base en la producción sostenible puede garantizar una mejor calidad de vida para la población rural. Este relato de experiencia busca exponer las percepciones obtenidas a través del trabajo desarrollado con un grupo de agricultores agroecológicos, residentes en una comunidad del área rural del municipio de Cacoal-RO. El objetivo es reportar una experiencia cuyos datos fueron recolectados a través de la experiencia y la observación sobre la influencia que las prácticas agroecológicas tuvieron en mejorar el bienestar de los agricultores y contribuir así a futuras investigaciones que se sumen a la capilaridad de las acciones agroecológicas. El grupo de agricultores está compuesto por 10 familias que trabajan directamente con la agroecología y participan activamente en las actividades. El trabajo de seguimiento del grupo fue de aproximadamente 12 meses. Las percepciones analizadas en este informe ocurrieron simultáneamente con el proceso de sistematización de prácticas agroecológicas del grupo Bem Viver, actividades que formaron parte de la ejecución de un proyecto de 
maestría. Se concluye que el trabajo de la huerta tuvo una fuerte influencia en la mejora de la autonomía y el bienestar de los miembros del grupo.

Palabras clave: Agroecología; Sistematización de la experiencia; Bienestar del horticultor rural.

\section{Introdução}

O modelo de desenvolvimento econômico atual induz e impõe transformações no modo de vida do trabalhador rural, influenciando diretamente na sua qualidade de vida, neste contexto estão submetidos a riscos de contaminação por agrotóxicos, entre outros (Pessoa \& Rigoto, 2012). Essa visão distorcida de desenvolvimento com ênfase nos métodos tradicionais de cultivo, como "única alternativa de progresso" desrespeita a vida e o ecossistema, ferindo os valores de povos (Porto \& Milanez, 2009). Um avanço em questão de compreender a saúde e sua relação direta com o meio ambiente é indispensável nesta situação, numa lógica que incorpore a dimensão socioambiental (Scopinho,2010).

Presume-se que os padrões de saúde e doença de agricultores rurais são decorrentes das condições de vida em que estão submetidos, que podem estar condicionados à fatores sociais, econômicos, tecnológicos e organizacionais relacionados ao perfil de produção e consumo, além de fatores de risco de natureza física, química, biológica, mecânica e ergonômica presentes nos processos de trabalho. No Brasil, aproximadamente 30 milhões de trabalhadores estão submetidos a riscos e agravos das condições de trabalho agrícola, equivalente a cerca de $20 \%$ da população economicamente ativa do país (Moreira, et al., 2015).

É neste contexto que a agroecologia com a sua perspectiva ecológica e social, surge como um fulgor à vida de agricultores rurais, a sua base em produção sustentável pode garantir uma maior qualidade de vida a população rural (Moreira, et al., 2015). Ressaltamos que as teias de relações "produção/trabalho, ambiente e saúde emaranhados pelo modo de produção e consumo são referências para se conhecer os riscos que todos estão expostos. Logo compreendendo a relação de condições de vida de grupos sociais (trabalhadores rurais) e condições ambientais de trabalho é o princípio racional para garantir vida e saúde para o ambiente e população (Dias, et al., 2006).

O adensamento e a difusão das experiências agroecológicas têm ampliado a visão sobre sua importância e propiciado um ambiente social e político adequado ao intercâmbio de inúmeras articulações e redes de modo a contribuir com o do desenvolvimento rural sustentável. Assim os benefícios da ciência agroecológica só serão alcançados quando houver uma expansão consistente dessas experiências (Holliday, 2006).

Daí a importância da sistematização de experiência que pode ajudar a responder os enigmas e desafios na atualidade, principalmente pelo seu caráter reflexivo e crítico somado ao compartilhamento de aprendizagem durante o processo. Para Sanches (2011), a sistematização de experiências vem conquistando espaços e a sua importância está na necessidade de tornar visíveis aquelas experiências que despontavam como resistência ao modelo de desenvolvimento rural. O que se busca, são experiências que servem de aprendizado e tragam desenvolvimento. Ao sistematizar tem-se uma interpretação do contexto da experiência, e a partir daí há um compartilhamento de aprendizagens, identifica e constrói abordagens teóricas para o aprimoramento das práticas e futuras pesquisas neste âmbito.

Esse relato de experiência implica na relevância da sistematização no campo agroecológico da agricultura familiar e traz resultados de um projeto sistematizador cujo objetivo inicial era sistematizar práticas agroecológicas que obteve ótimos resultados, mas também abriu-se leques para o desenvolvimento de futuras pesquisas neste contexto, que é principal objetivo desse relato, de forma a aumentar a capilaridade das ações agroecológicas. Além disso o projeto sistematizador demostrou ser ferramenta transdisciplinar demostrando como a sistematização de práticas agroecológicas veio a contribuir com o bem-estar dos horticultores. 


\section{Relação Agroecologia, Sistematização de Experiência e Bem Estar de Trabalhadores Rurais}

No processo de resistência ao modo de vida, se insere as lutas com base na produção de conhecimento e estímulo a uma práxis contrária à lógica do agronegócio. Neste contexto a agroecologia como ciência busca integrar os saberes históricos dos trabalhadores do campo com os conhecimentos de diferentes ciências (Caporal \& Azevedo, 2011. Guzmán, Molina \& Sevilla, 2000).

Alguns estudos relacionados ao processo saúde-doença demonstram que essa relação é influenciada pela forma de (re)produção dos trabalhadores rurais em articulação com a sociedade. Pressupõe-se que a dependência de um conjunto de técnicas introduzidas por uma lógica externa capitalista que favorece o uso de agrotóxicos é determinante no adoecimento por meio deles. Assim como um ambiente influenciado pelos princípios da agroecologia confere mais autonomia ao processo de trabalho, relação com o movimento social, o consumo da produção, a maior preocupação com o ambiente e com a qualidade da alimentação da população (Lira, et al., 2018).

Neste cenário a compreensão dos agrotóxicos enquanto relevante problema ambiental e de saúde pública vem crescendo em paralelo à ampliação de seu uso e das evidências dos impactos que podem causar, no estado de Rondônia de acordo com último censo realizado houve um notório crescimento de contaminação para a agricultura familiar, a incidência de notificações por intoxicações cresceu acompanhando a taxa de consumo (Plano Estadual de Vigilância em Saúde de Populações Expostas a Agrotóxicos do Estado de Rondônia, 2013).

Analisando os percursores históricos sobre o uso de agrotóxico utilizado em demasia pela agricultura convencional, podemos perceber as diferentes etapas pelas quais passou a utilização desses produtos, neste contexto também podemos compreender a influência que a vivência prática de técnicos e agricultores teve sobre a utilização e a percepção desses agrotóxicos ao longo de 50 anos. A partir dessa análise, existem algumas evidências para sugerir que o uso e a percepção dos agrotóxicos dependerão não só de estudos técnicos sobre o seu impacto na saúde e no meio ambiente, mas também de concepções e experiências individuais de agricultores, técnicos, políticos e ambientalistas (Carvalho, et al., 2017).

Somando a estas questões através de algumas experiências verifica-se que a agricultura agroecológica revela-se como ferramenta promotora de saúde por contribuir com o fortalecimento do protagonismo individual e comunitário, empoderamento, criação de ambientes favoráveis à saúde, bem como seu potencial para a multiplicação de "saberes", pelos envolvidos, sobre temas relacionados à geração de renda, saúde e meio ambiente (Ribeiro, et al., 2015)

Há demonstrações que através de operacionalização teórico-prática, foram desenvolvidos constructos subjetivos, com a sustentação da viabilidade da agroecologia como modelo de produção para a melhoria das condições de vida e saúde nas propriedades e comunidades rurais, assim como a elaboração de instrumentos que vem orientando o amplo processo de transição agroecológica e de organização camponesa (Abreu \& Alonzo, 2018).

Reforçando todos esses argumentos, a compreensão do processo de sistematização de experiências neste contexto é o princípio para se fazer um bom trabalho, dele se depreende uma metodologia de pesquisa qualitativa evidenciando a participação, que tem por meta levar os envolvidos a olhar para iniciativas diferenciadas de desenvolvimento rural, interpretando e utilizandoas como instrumento de aprendizagem. No campo da ciência que se utilizam de sistematização de experiências as iniciativas são inovadoras com enorme potencial de aprendizado, onde o processo parte da prática, da realidade para a teorização e partir de então além do aprendizado, o que não é comum aos outros tipos de pesquisa. Os projetos de sistematização também servem de referências para programas, projetos, políticas públicas e ações de extensão (Cidac \& Holliday, 2007).

\section{Metodologia}

Este artigo configura como um relato de experiência, em que são descritos resultados inerente de etapas oriundas do projeto de sistematização de práticas agroecológicas em núcleos de agricultura familiar do município de Cacoal-RO denominado 
grupo Bem Viver, as famílias que compõem o grupo se localizam a uma distância entre 30 e 40 quilômetros da zona urbana, somando uma comunidade de 10 famílias. Esta pesquisa se caracteriza por ser qualitativa e descritiva, de caráter exploratório. Do projeto participaram todos as pessoas das familias que trabalhavam diretamente com as atividades da horta.

Figura 1. Mapas de localização grupo Bem viver, RO-Brasil.

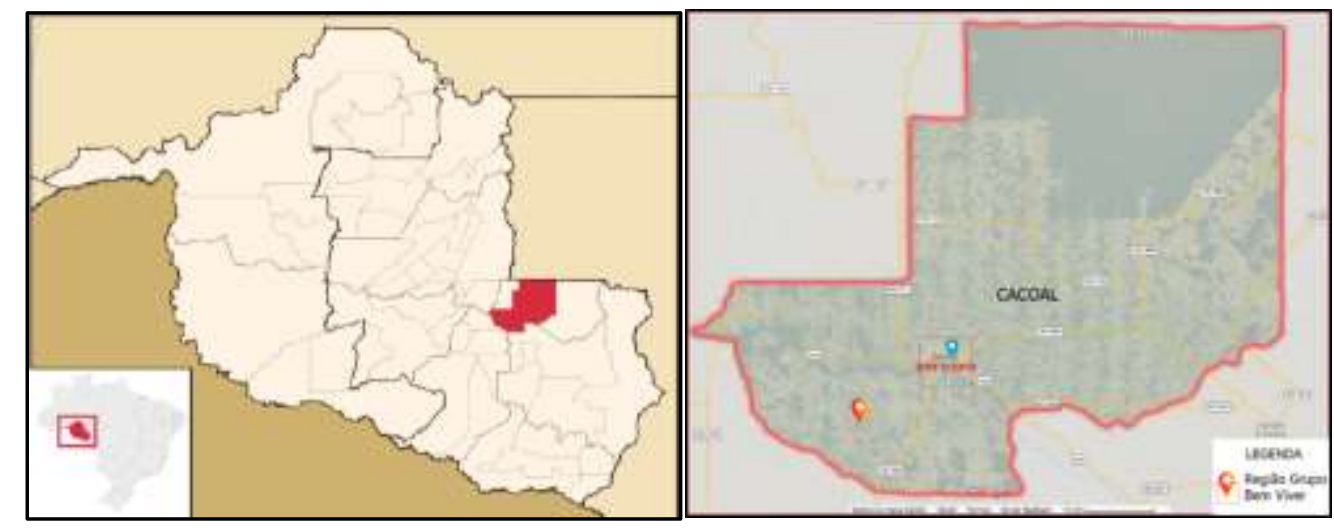

Fonte: google maps. Organização Fernanda da Silva Pereira.

A metodologia de sistematização foi flexível e embasada em alguns critérios que podem ser chamados de fases. Esse processo possui quatro dimensões que se inter-relacionam mutualmente:

Análise - o que aconteceu. Quem se envolveu.

Síntese - o que resultou.

Indução - qual o diferencial dessa experiência?

Dedução - quais aprendizagens podem ser referência pra outras experiências.

Além disso, para Holiday (2006) o método coerente deve considerar os cinco pontos pelo qual o processo se guia, cujo foram feitas as adaptações (Quadro 1). Conforme foi desenvolvendo cada fase do projeto, os dados foram coletados simultaneamente através das diversas experiências particulares dos agricultores, que aconteceu através da vivência, do diálogo e coleta de informações (entrevistas semiestruturadas), no decorrer de 12 meses. Com base na avalição do processo vivido foi possível fazer uma relação entre a influência das práticas agroecológicas com o bem-estar dos agricultores durante o processo sistematizador.

Com finalidade de situar o leitor, o projeto foi a base para obtenção dessas informações e compreendeu algumas etapas. 
Quadro 1. Ações interdisciplinares desenvolvidas no projeto.

\begin{abstract}
O ponto de partida, onde foi definido grupo agroecológico a ser trabalhado, números de famílias; Contato, participação e registros iniciais.

As Perguntas iniciais, definição do eixo de sistematização, que foram manejo de solo e diversificação de cultura, nesta etapa também foram feitas as visitas técnicas, acompanhamento e estudo local com base nos processos mais carentes de informação e tecnologias da comunidade. Adoção de entrevista semiestruturada como técnica de coleta, para a captação de dados subjetivos, utilizando roteiros previamente elaborados. Acompanhamento semanal e todas as informações anotadas em formulários e confecção de relatórios das atividades. Também teve acompanhamento diário das atividades e reunião com cada produtor para discutir o processo de produção. Questionário que auxiliou na interpretação do processo. Planejamento de reuniões.
\end{abstract}

Recuperação do processo vivido: comparação do antes e depois do processo de sistematização; Identificar os objetivos de aprendizagem; Motivação inicial dos agricultores; Principais ações realizadas; Conteúdos trabalhados e por que; Evolução que obtiveram quanto aos aspectos de produção, econômicos e sociais; Dificuldades encontradas; Formas de participação existentes e novas participações geradas; Opiniões e concepções dos produtores sobre.

Reflexão de fundo: reconstrução histórica, detalhada, conjuntamente com as atividades formativas, as características de cada sistema produtivo, ressaltado as suas diferenças e peculiaridades, uma vez que serão decisivos para contribuir para a experiência de cada um deles.

Pontos de chegada: Formulação de conclusões e resultados concretos da articulação entre os diferentes grupos e os envolvidos. Formulação de propostas para que as experiências sejam compartilhadas, para retroalimentarse. Comunicação de aprendizado.

Fonte: Autores (2020).

\title{
4. Resultados e discussão
}

No desenvolver do projeto sistematizador o principal objetivo era realizar a sistematização de práticas agroecológicas do Grupo Bem Viver com a finalidade de fortalecer o processo de transição agroecológica e contribuir com a disseminação de conhecimentos, especificamente trabalhando melhoria nos sistemas produtivos com relação as práticas agroecológicas desenvolvidas dentro da horta e consequentemente produzir comais qualidade, tendo uma maior eficiência nos seus sistemas produtivos. Contudo no decorrer da experiência surgiram informações importantes que foram além dos objetivos almejados pelo projeto, sendo possível fazer essa relação da experiência com o bem-estar dos horticultores. Ressaltamos aqui a importância do projeto sistematizador que conseguiu ter um maior alcance das suas ações em benefícios de trabalhadores rurais.

Durante a vivência, que aconteceu através do diálogo, de entrevistas informais, das reuniões, visitas técnicas e a reconstrução histórica foi possível analisar que o trabalho da horta se tornou primordial com o passar do tempo para restabelecer a autonomia e bem-estar dos integrantes do grupo.

No total a base do grupo Bem viver é formada por 28 pessoas que fazem parte de unidade familiar, através dos relatos $2 \%$ das pessoas disseram ter melhora de seus problemas psicológicos depois de trabalhar na horta agroecológica. Também foi possível verificar durante o tempo de vivência a melhora da autoestima das pessoas envolvidas no projeto, essas análises foram percebidas através da maior autonomia em representatividade nas reuniões mensais e com relação ao próprio trabalho na horta. 
Podemos verificar que o processo sistematizador de práticas agroecológicas ofereceu um caminho aos estudos da saúde e de processos sociais que devem ser investigados com maior profundidade.

\section{Considerações Finais}

Através da sistematização de experiência, voltada para as práticas agroecológicas do grupo Bem Viver foi possível estabelecer uma conexão de como o trabalho em hortas agroecológicas influenciou na recuperação de problemas associados ao bem-estar dos agricultores (depressão, traumas semelhantes, melhora da autoestima etc.). Nota-se como o trabalho interdisciplinar é percebido e vivenciado como sendo de extrema importância para uma atenção a uma maior qualidade de vida. Que a aproximação da teoria, prática e seus desafios caminham juntos na construção do saber, empoderamento, participação e identidade.

Diversos temas para o avanço da pesquisa agroecológica surgiram atualmente, isso porque há a necessidade de expandir as escalas espaciais e temporais das investigações agroecológicas, bem como utilizar métodos diferenciados e flexíveis, de modo que os resultados possam ser sintetizados de maneira mais efetiva. Também se tem um apelo por abordagens que que utilizam as dimensões humanas da agroecologia. O avanço da ciência agroecológica e dos sistemas alimentares sustentáveis depende de investimentos em pesquisa agroecológica, bem como de cuidadosa consideração de fatores sociais, políticos e culturais que impedem uma adoção mais ampla.

Diante disso ressalto a importância do processo sistematizador que auxiliou no aspecto de compartilhar conhecimento das práticas realizadas, mas também permitiu identificar aspectos relacionados à saúde dos trabalhadores que devem ser investigados com profundidade e devida importância. Deixo aqui sugestões de pesquisas que aprofundem na relação da saúde de agricultores e seu modo de produzir, incluindo projetos de sistematização focado na melhoria e bem-estar de agricultores/horticultores. Esses trabalhos devem ser realizados nos diversos núcleos de agroecologia existentes.

A sistematização de experiência se torna notória e aponta caminhos para que os aprendizados contidos nas práticas cotidianas e desenvolvidas por um montante de atores rurais que participam do processo possam contribuir com o fortalecimento do campo agroecológico. Se torna um exercício rigoroso de aprendizagem e interpretação crítica dos processos vividos e auxilia em recriar as práticas e renovar a produção teórica da ciência a partir das experiências. Também pode ampliar os debates de conservação dos recursos naturais e consciências dos efeitos do uso de agrotóxicos.

Embora se tenha consciência da importância do processo dinâmico e construtivo da sistematização de experiência, precisamos mudar a ideia que o processo sistematizador é difícil de ser implementado, para que os objetivos da agroecologia se cumpra, dando prioridade institucional a sistematização de experiência. Reforçamos a inexistência de fórmulas e receitas para fazer a sistematização, que deve ser totalmente flexível para atender a complexidade e especificidade de cada grupo local trabalhado. Em dado momentos o processo sistematizador potencializa a capacidade dos grupos para se auto representar e para representar a própria experiência.

\section{Referências}

Abreu, P. H. B., \& Alonzo, H. G. A (2018). Salutogênese-Camponês a Camponês: uma metodologia para promoção da saúde de populações expostas a agrotóxicos. Relato de experiência. Case study. Saúde debate. Rio de janeiro, 42 (4), 261-274.

Caporal, F. R., \& Azevedo, E. O. (2011). Princípios e perspectivas da agroecologia. Instituto Federal de Educação, Ciência e Tecnologia do Paraná - Educação a Distância.

Carvalho, M. M. X., Nodari, E. S., \& Nodari, R. O. (2017). "Defensivos” ou "agrotóxicos"? História do uso e da percepção dos agrotóxicos no estado de Santa Catarina, Brasil, 1950-2002. Hist. cienc. Saúde Manguinhos, Rio de Janeiro, 24 (1), 75-91.

Cidac (Centro de Informação e Documentação Amílcar Cabral), \& Holliday, O. J. (2007). Sistematização de experiências: aprender a dialogar com os processos. 74 .

Dias, E. C. (2006). Condições de vida, trabalho, saúde e trabalho dos trabalhadores rurais no Brasil. Saúde do trabalhador rural, RENAST. 
Research, Society and Development, v. 10, n. 2, e2710212067, 2021

(CC BY 4.0) | ISSN 2525-3409 | DOI: http://dx.doi.org/10.33448/rsd-v10i2.12067

Guzman Casado, G., González de molina, M., \& Sevilla Guzmán, E. (2000). Introducción a la Agroecología como desarrollo rural sostenible: Ediciones MundiPrensa.

Holliday, O. J. (2006). Para sistematizar experiências. Tradução de: Maria Viviana V. Resende. (2a ed.) 128 p.

Lira, P. R. A., Albuquerque, P. C. C., \& Gurgel, I. G. D. (2018). Trabalho e estranhamento: a determinação social da saúde em assentamentos. Trab. educ. saúde, Rio de Janeiro, 16 (2), 431-452.

Moreira, J. P. L., Oliveira, B. L. C. A., Muzi, C. D., Cunha, C. L. F., Brito, A. S., \& Luiz, R. R. (2015). A saúde dos trabalhadores da atividade rural no Brasil. Cad. Saúde Pública, Rio de Janeiro, 31(8), 1698-1708.

Pessoa, V. M., \& Rigoto, R. M. (2012). Agronegócio: geração de desigualdades sociais, impactos no modo de vida e novas necessidades de saúde nos trabalhadores rurais. Rev. bras. saúde ocup, São Paulo, 37(125), 65-77.

Plano Estadual de Vigilância em Saúde de Populações Expostas a Agrotóxicos do Estado de Rondônia. (2013). Secretaria de Estado da Saúde Agência Estadual de Vigilância em Saúde.

Porto, M. F., \& Milanez, B. (2009). Eixos de desenvolvimento econômico e geração de conflitos socioambientais no Brasil: desafios para a sustentabilidade e a justiça ambiental. Ciênc. saúde coletiva, Rio de Janeiro, 14(6), 1983-1994.

Porto. M. F., \& Soares, W. L. (2012). Modelo de desenvolvimento, agrotóxicos e saúde: um panorama da realidade agrícola brasileira e propostas para uma agenda de pesquisa inovadora. Rev. bras. Saúde ocup, São Paulo, 37 (125), 17-50.

Ribeiro, S. M., Bogus, M., \& Watanabe, H. A. W. (2015). Agricultura urbana agroecológica na perspectiva da promoção da saúde. Saúde soc, 24 (2), $730-743$.

Sanches, C. D. A. (2011). A contribuição da sistematização de experiência para o fortalecimento do campo agroecológico e agricultura familiar no Brasil. São Carlo- UFScar, $180 \mathrm{p}$.

Scopinho, R. A. (2010). Condições de vida e de saúde do trabalhador em assentamento rural. Ciência \& Saúde Coletiva, Rio de Janeiro, 15(1), $1575-1584$. 